\title{
Effect of start-stop motion on contact replenishment in a grease lubricated deep groove ball bearing
}

\author{
Hui Cen ${ }^{\text {a,*, Piet M. Lugt }}{ }^{\text {b, } c}$ \\ ${ }^{a}$ School of Electrical and Mechanical Engineering, Xuchang University, Xuchang, Henan, 461000, PR China \\ ${ }^{\mathrm{b}}$ SKF Research and Technology Development, Houten, the Netherlands \\ ${ }^{\mathrm{c}}$ University of Twente, $7500 \mathrm{AE}$, Enschede, the Netherlands
}

A R T I C L E I N F O

\section{Keywords:}

Start-stop

Grease lubrication

Ball bearing

Replenishment

\begin{abstract}
A B S T R A C T
The impact of start-stops on the thickness of lubricating films in the Elasto-Hydrodynamic Lubrication (EHL) contacts in grease lubricated rolling bearings is different from that in oil lubricated bearings. This paper shows that the film thickness drops to zero quickly due to the absence of hydrodynamic effects during a stop. It quickly increases to an unexpected large value when the bearing is stopped for a long time and started up again, although this large value only lasts for a very short time. This is due to additional track replenishment during the stop leading to an initially thicker film than expected. This increase is found to be determined by the rotational speed of the bearing, stop-time, base oil viscosity and width of the contacts. The start-stop effect is similar for unidirectional and oscillating speeds, provided that the stopping time is long enough.
\end{abstract}

\section{Introduction}

In a rolling bearing, the lubricating grease is supposed to separate the rolling elements from the rings by means of lubricating films. These films can be calculated in the fully flooded regime by applying the base oil viscosity with the empirical equations developed by Hamrock and Dowson [1] or Nijenbanning [2]. However, bearings lubricated with grease are mainly working under starved lubrication conditions where the supply of oil to the inlet of the contacts is dominating the thickness of the lubricating film. We earlier showed that this supply is a local phenomenon [3]. It is provided by a flow from the "grease reservoir" next to the running tracks. So the replenishment mechanism is based on flow where the supplied lubricant volume to the inlet of the contacts is clearly a function of the bearing speed, which translates into an available time for replenishment. The time for replenishment decreases with increasing speed resulting in more starvation. This has been investigated quite extensively in earlier work for single or multiple contacts [4-17] and full bearings [3,18-21]. It is shown in the authors' previous paper [3] that the thickness of the lubricating film itself does not always decrease with increasing speed. An increasing speed reduces the time for side flow in the individual contacts as well [16]. Both effects seems to (at least almost) compensate each other.

For single or multiple contacts, the optical interferometry method is the most commonly used and most accurate method to measure the film thickness. This method has been applied by many researchers in ball-ondisc rigs to study the starvation behavior in elasto-hydrodynamic lubrication (EHL). Wedeven et al. [4] found that the starvation of grease lubricated contacts could be attributed to the transportation and redistribution of lubricant in the vicinity of the inlet region. Chiu [5] attributed starvation to the lack of track replenishment from the oil layers next to the rolling track during the time interval between over-rollings. Wen et al. [6] stated that, if the local stress exceeds the yielding shear stress of the tested grease in line contacts, a non-axial flow region would form and result in a steady EHL film. Chevalier [7] showed that the thickness of lubricating film is dominated by the oil layers feeding the contacts, rather than the meniscus position. Both are difficult to measure or predict. Jacod et al. [8] found that replenishment in a starved EHL contact happens locally, rather than "in-between-over-rollings", a concept that was proven by the calculations from Gershuni et al. [22]. Cann [9-11] and her co-authors showed that local replenishment in greased lubrication can be induced by ball spin, capillary forces, vibrations or variable loads. In the models of Damiens et al. [13] and Van Zoelen et al. [14-16], the lubricant layer at the outlet region within a contact acts as an inlet layer to the next contact, assuming no replenishment, a concept that was experimentally illustrated by Svoboda et al. [17] who used an optical test apparatus with multiple

\footnotetext{
* Corresponding author.

E-mail address: hui.cen@foxmail.com (H. Cen).
} 
contacts to perform measurements of the starved EHL thickness in a ball/disc contact positioned after a roller/disc contact.

For film thickness measurements in full bearings, the electrical capacitance approach is the most commonly used technique, which has also been extensively used to study starvation. Wikström and Jacobson [18] applied this method in a spherical roller bearing lubricated with greases and suggested a feed-loss mechanism to explain the fact that the lubrication condition in grease lubricated bearings is less starved than expectations based on starved EHL theory only. Here the oil supply to the contacts is caused by grease bleeding and replenishment by capillary forces or by centrifugal forces. Lubricant loss may be caused by oxidation, polymerization, evaporation, centrifugal force induced thin film flow or droplet formation in the outlet of the contacts. An extensive description of these mechanisms can be found in Ref. [23]. Zhou et al. [19] showed that the thickness of the lubricating film at medium speeds in a ball bearing lubricated with greases under starved lubrication is not only related to the viscosity of the corresponding base oil but also depends on replenishment, which is determined by bleed, mechanical stability and mobility of the grease. In a previous paper published by the authors [20], the thickness of the lubricating film within the grease lubricated contacts in a ball bearing was found to be around $1 / 4$ of that calculated with the base oil viscosity under fully flooded conditions after $20 \mathrm{~h}$ of continuous running, i.e. well after the churning phase. In the authors' last paper [3], it is shown that the starved film thickness in an axially loaded bearing, lubricated with grease, after $20 \mathrm{~h}$ running, is only affected by the product of viscosity of base oil, bearing rotation speed and width of the contacts. This shows that replenishment in a real bearing is a local contact phenomenon. The measurements showed that the only relevant physical property that determines the film thickness at this early stage in the early lifetime of a grease is the base oil viscosity, so not the bleed rate or any grease rheological properties. We also showed in this paper that the speed parameter has limited effect on the film thickness.

In all papers referred to above, the speed was kept constant during a test. However, in most applications bearings are not running continuously. Models for grease life are based on constant conditions, whereas in most applications bearings are running under varying conditions. Calculations are then done using the Palmgren-Miner rule [24]. Here it is assumed that the "damage" is load history independent. So, grease life is assumed to be equal for a bearing running under high followed by low speed conditions and for a bearing running under low speed and then high speed.

Much speculation exist around the potential damage of bearings with starts and stops. Earlier work was done by Cann and Lubrecht with the optical ball on disk apparatus [10], where they stopped the rotation for $20 \mathrm{~min}$ and start the rotation again. The film thickness immediately after the stop was found to be larger than it was before the stop, but decayed to a similar level as in the equilibrium state. This was a direct prove of the occurrence of contact replenishment by the lubricant in starved contacts. So far nothing has been published on these phenomena that may also occur in full bearings.

For oil lubricated bearings start-stops are regarded as unfavorable because the film thickness will be zero at zero-speed, which may damage the bearing. In the case of starved lubrication, such as in grease lubricated bearings, this may be totally different.

We will show that start-stops may actually be favorable for replenishment. This is done by studying the film thickness in a full bearing. This replenishment time will of course be affected by various operating conditions (e.g. load, speed) as well as grease type. In this paper we therefore conducted start-stop experiments showing the impact of startstops on film thickness. We will systematically vary the conditions to show the general behavior and the impact of the various parameters on the replenishment rate.

\section{Experimental}

\subsection{Description of bearing test apparatus}

The bearing test apparatus in this paper is equipped with a "Lubcheck" instrument that measures the bearing electrical capacity. It uses a high frequency capacitive voltage divider to reduce the noise level of the measured signals. Details can be found in Heemskerk et al. [25]. The electrical capacitance is determined by the distance between the rolling elements and raceways, i.e., the lubricating films. Translating the measured electrical capacitance into a film thickness is not trivial. For a theoretical calculation, values for various physical parameters such as the dielectric constant of the lubricant under high pressure need to be known. Here an empirical methodology was used where the relation between capacitance and film thickness was established using measurements with base oil where the bearing was running under full film conditions. A pure axial load was applied so that the thickness of the lubricating film can be assumed to be equal for all contacts. With a fixed outer ring of the test bearing, an electrical motor with a magnetic coupling was applied to drive the inner ring. The overall appearance and the principle of the test rig is shown in Fig. 1 and Fig. 2. The electrical capacitance of the test bearing was measured and then converted into film thickness by applying the calibration method developed by Wilson [26]. More details of the test rig, calibration tests and test procedures can be found in a previous paper [20].

\subsection{Materials and test conditions}

A standard ball bearing with designation 6209-2Z/C3 was tested in this paper. Three different greases, $\mathrm{PU} / \mathrm{E}, \mathrm{Li} / \mathrm{M}$ and $\mathrm{LiC} / \mathrm{PAO}$, were tested separately with a single bearing. Details of the greases and test bearing are shown in Table 1 and Table 2 . The constant pure axial loads that were used were 513, 700 and $900 N$ (C/P: 34.1, 26.4 and 21.8), resulting in inner ring contact pressures $p_{\max }$ of $1.39,1.54,1.67 \mathrm{GPa}$, respectively. The speeds of the inner ring of the tested bearings were controlled at 1000, 1500, $2500 \mathrm{rpm}$, resulting in $\mathrm{ndm}$ (speed $(\mathrm{rpm}) \times$ mean bearing diameter $\left(d_{m}=65 \mathrm{~mm}\right)$ ) ranged from 65,000 to 162,500 . A thermocouple embedded in the bearing housing, in contact with the stationary outer ring of the bearing was applied to record the (selfinduced) temperature. Test bearings were first run at a fixed speed for $20 \mathrm{~h}$ (to ensure that the bearing is running in the semi-steady state bleeding phase), followed by stops of $5 \mathrm{~s}, 1 \mathrm{~min}, 5 \mathrm{~min}, 10 \mathrm{~min}, 30 \mathrm{~min}$, $1 \mathrm{~h}, 2 \mathrm{~h}$ and $13 \mathrm{~h}$. After each stop, the speed was set back to the original speed. The load was fixed, which means that the bearing was under the same load throughout the test, even during the stops. Fig. 3 shows a sample result of the thickness of lubricating film and temperature throughout the whole test. The temperature is self-induced and therefore varies. To eliminate this effect, the normalized film thickness, $h_{g} / h_{f f}$, is plotted. Here $h_{g}$ is the thickness measured within the grease lubricated contacts and $h_{f f}$ is the calculated thickness of lubricating film by applying viscosity of base oil with the Hamrock Dowson equation [1], where this viscosity was corrected with the temperature measured on the outer ring, using Walther's equation [27]. One may argue on the accuracy of the base oil film thickness $h_{f f}$ obtained from Hamrock Dowson equation. However, this paper only introduced $h_{f f}$ to obtain a normalized film thickness $h_{g} / h_{f f}$, to eliminate the effect of varying temperature in each test. The normalized film thickness first increased after each stop but subsequently dropped back to the same level as before the stop.

\section{Measurements}

\subsection{Varying speed}

Film thickness measurements were performed at a constant load of $513 N$ with the three grease samples at speeds of 1000,1500 and 2500 


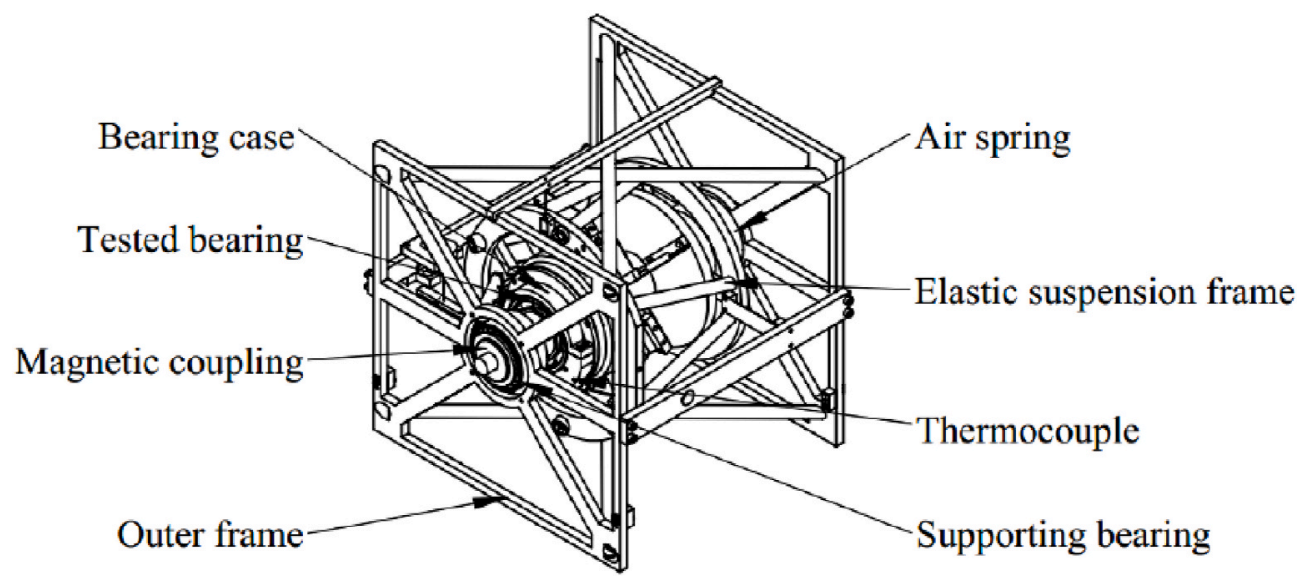

Fig. 1. Overall appearance of the bearing test apparatus.

\section{Outer ring}
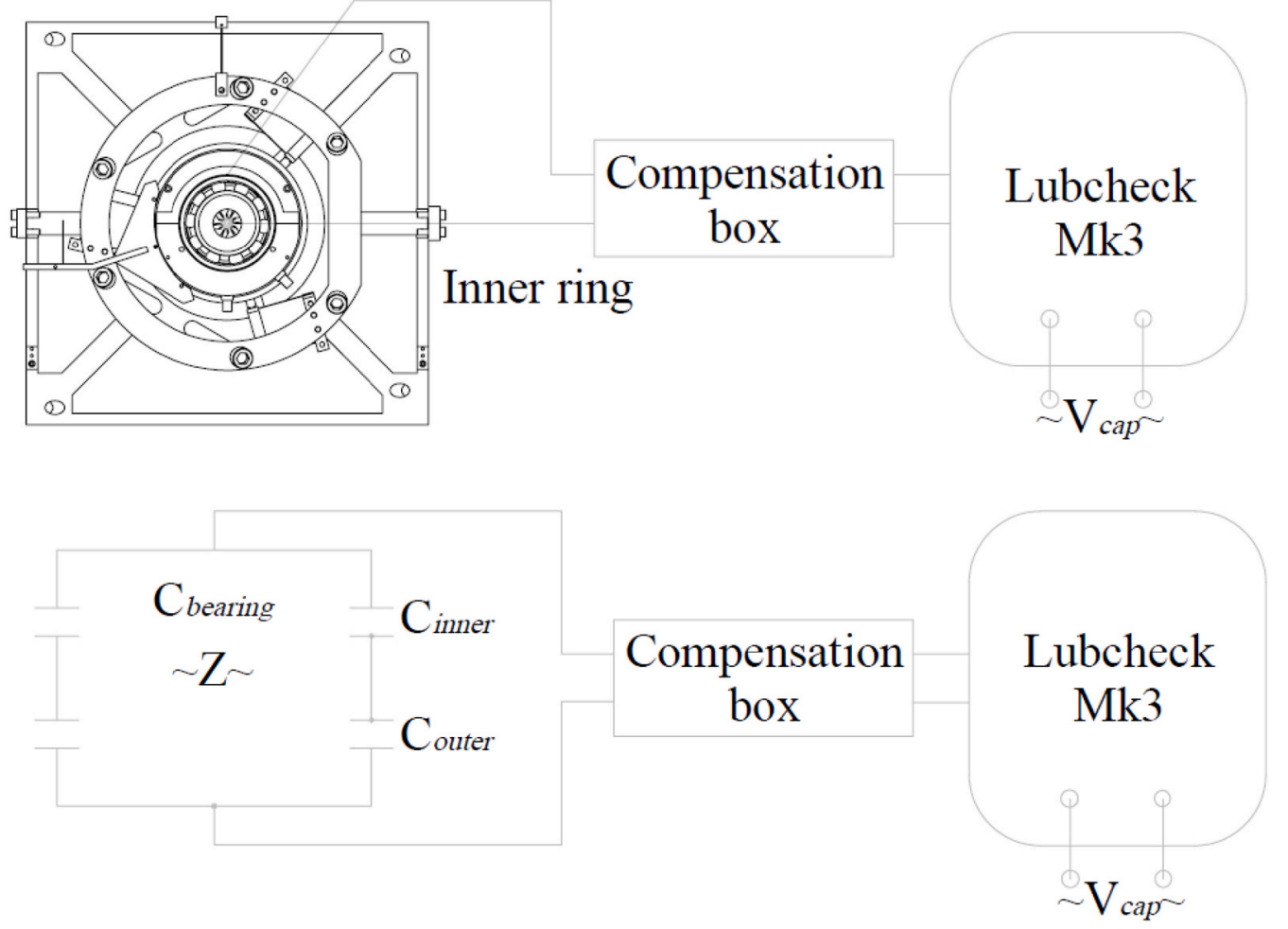

Fig. 2. Principle of the bearing test rig where the gaps (film thickness) between balls and rings are forming two capacitors with capacitance $\mathrm{C}_{\text {inner }}$ and $\mathrm{C}_{\text {outer }}$.

Table 1

Test grease properties.

\begin{tabular}{|c|c|c|c|c|c|c|c|}
\hline $\begin{array}{l}\text { Grease } \\
\text { designation }\end{array}$ & NLGI & $\begin{array}{l}\text { Base oil } \\
\text { type }\end{array}$ & $\begin{array}{l}\text { Thickener } \\
\text { type }\end{array}$ & $\begin{array}{l}\text { Base oil viscosity } \\
40^{\circ} \mathrm{C}(\mathrm{cSt})\end{array}$ & $\begin{array}{l}\text { Base oil viscosity } \\
100^{\circ} \mathrm{C}(\mathrm{cSt})\end{array}$ & $\begin{array}{l}\text { Density of base oil }(\mathrm{g} / \\
\left.\mathrm{cm}^{3}\right) \text { at } 25^{\circ} \mathrm{C}\end{array}$ & $\begin{array}{l}\text { Base oil pressure-viscosity coefficient } \alpha \\
\left(10^{-9} \mathrm{~Pa}^{-1}\right) \text { at } 25^{\circ} \mathrm{C}\end{array}$ \\
\hline $\mathrm{PU} / \mathrm{E}$ & $2-3$ & Ester & Polyurea & 70 & 9 & 0.91 & 24 \\
\hline $\mathrm{Li} / \mathrm{M}$ & 3 & Mineral oil & Lithium & 100 & 10 & 0.90 & 27 \\
\hline $\mathrm{LiC} / \mathrm{PAO}$ & $2-3$ & PAO & $\begin{array}{l}\text { Lithium } \\
\text { complex }\end{array}$ & 191 & 42 & 0.85 & 17 \\
\hline
\end{tabular}

rpm. Fig. 3 shows that the normalized film thickness becomes quite stable after running for $20 \mathrm{~h}$, confirming that the bearing is running in the bleed phase [23]. After each stop-start, the normalized film thickness first increased and then decreased to a similar value as the stable thickness at the end of the first $20 \mathrm{~h}$. Hence, after a stop, track replenishment takes place, such that the contacts are running less starved when starting up again. The level of replenishment is clearly a function of the time that the bearing was standing still. The figure shows that stopping for $1 \mathrm{~h}$ was enough to provide full replenished running tracks again. Fig. 4 presents the development of the normalized film 
Table 2

Properties of test bearing.

\begin{tabular}{|c|c|c|c|c|c|c|}
\hline $\begin{array}{l}\text { Bearing } \\
\text { dimensions }\end{array}$ & $\begin{array}{l}\mathrm{d}_{\mathrm{a}} \\
(\mathrm{mm})\end{array}$ & $\mathrm{d}_{\mathrm{m}}(\mathrm{mm})$ & $\begin{array}{l}\mathrm{d}_{\mathrm{i}} \\
(\mathrm{mm})\end{array}$ & $\begin{array}{l}\mathrm{d}_{\mathrm{b}} \\
(\mathrm{mm})\end{array}$ & $\mathrm{d}_{0}(\mathrm{~mm})$ & $\mathrm{d}(\mathrm{mm})$ \\
\hline & 85 & 65 & 57.6 & 45 & 72.4 & 12.3 \\
\hline \multirow[t]{2}{*}{$\begin{array}{l}\text { Contact } \\
\text { geometries }\end{array}$} & $\begin{array}{l}R_{x 1} \\
(\mathrm{~mm})\end{array}$ & $R_{x 2}(\mathrm{~mm})$ & $\begin{array}{l}R_{y 1} \\
(\mathrm{~mm})\end{array}$ & $\begin{array}{l}R_{y 2} \\
(\mathrm{~mm})\end{array}$ & & \\
\hline & 6.15 & 6.15 & 26.3 & 6.3 & & \\
\hline \multirow[t]{2}{*}{ Materials } & Code & $\begin{array}{l}\text { Roughness } \\
(\mathrm{nm})\end{array}$ & \multicolumn{2}{|c|}{$\begin{array}{l}\text { Number of balls, } \\
Z\end{array}$} & Clearance & \\
\hline & $\begin{array}{l}\text { AISI } \\
52100\end{array}$ & $<10$ & 10 & & $\mathrm{C} 3$ & \\
\hline
\end{tabular}

thickness during a start versus the stop time, for different speeds. This start film thickness $h_{g} / h_{f f}$ (denoted as highest $h_{g} / h_{f f}$ after the stop in the figure) was calculated by taking the average of the film thickness measurements in the first $10 \mathrm{~s}$ after a start. It can be seen that until about a stop time of $1 \mathrm{~h}$, the start $h_{g} / h_{f f}$ value after each stop interval increased with increasing stop time. At some stop time the contacts are fully flooded again and a further increase of the stop time will therefore not lead to thicker start films. The stop time to achieve fully flooded conditions is different for the different greases, which must be related to the mobility of bled oil for replenishment. In our earlier paper [3] we showed that the film thickness at this stage depends only on the base oil viscosity. Bleed, consistency etc. does not play a role for these greases. All this must become relevant at a much later stage but not until $24 \mathrm{~h}$ of running at these moderate temperatures.

Fig. 5 is a compilation of the results of Fig. 4, omitting the results where the film thickness has reached a steady value, where $t$ is the stop time (seconds), $u$ is the linear speed $(\mathrm{m} / \mathrm{s})$ and $\eta$ is the base oil dynamic viscosity at the ambient temperature. So this figure shows the results up to full replenishment. In this figure the increase in film thickness $\Delta h=$ $h_{g}-h_{g 0}$, is used as the variable on the ordinate, where $h_{g 0}$ is the film thickness before the stop. The parameter $\Delta h$ is a measure of the replenishment volume.

The replenishment volume (for this bearing and under this load) can be approximated by

$\Delta h \sim \frac{t^{1 / 2} u}{\eta^{0.2}}$

The viscosity changes during "the stop" where the bearing cools down in the absence of a frictional heat source. Therefore we have taken the average viscosity of the base oil during each stop. At this stage, it is difficult to separate the impact of viscosity increase (due to the temperature decrease during the stop) and replenishment on the increase of film thickness once the bearing is restarted.

The increase of film thickness $h_{g}-h_{g 0}$ by stopping increases with an increase of speed. This effect is even stronger than that of time or viscosity. This is surprising since replenishment is happening during standstill. However, the measured thickness of the lubricating film within the contacts -the EHL film - is not equal to the layer thickness of oil inbetween the contacts. This makes the interpretation not trivial. Before stopping, the bearing is running in starved lubrication conditions where we earlier measured [3]:

$h_{g} \sim \frac{1}{u^{0.12}}$

During starting up again, the contacts are less starved. In the extreme case, i.e. under fully flooded conditions:

$h_{g} \sim \frac{1}{u^{0.67}}$

This difference in speed dependence of the EHL film thickness explains also a speed dependence in the apparent replenishment $\Delta h$. However, the high exponent that is found here cannot really be explained.

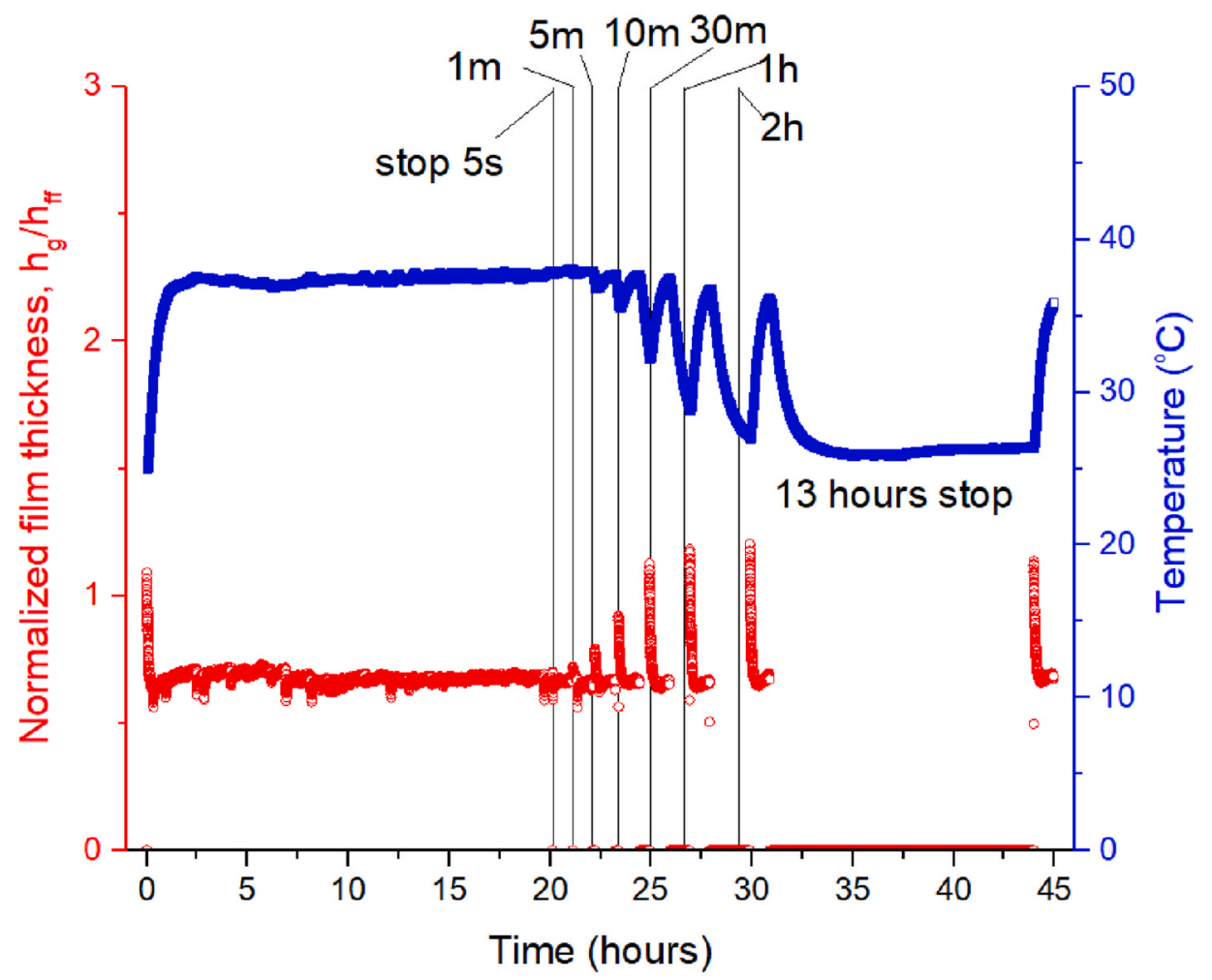

Fig. 3. Film thickness measurement results for Li/M at speed of $1000 \mathrm{rpm}$ and load of $513 \mathrm{~N}$. The bearing is stopped for $5 \mathrm{~s}, 1,5,10,30 \mathrm{~min}, 1 \mathrm{~h}, 2 \mathrm{and} 13 \mathrm{~h}$, and then re-started for $1 \mathrm{~h}$. 

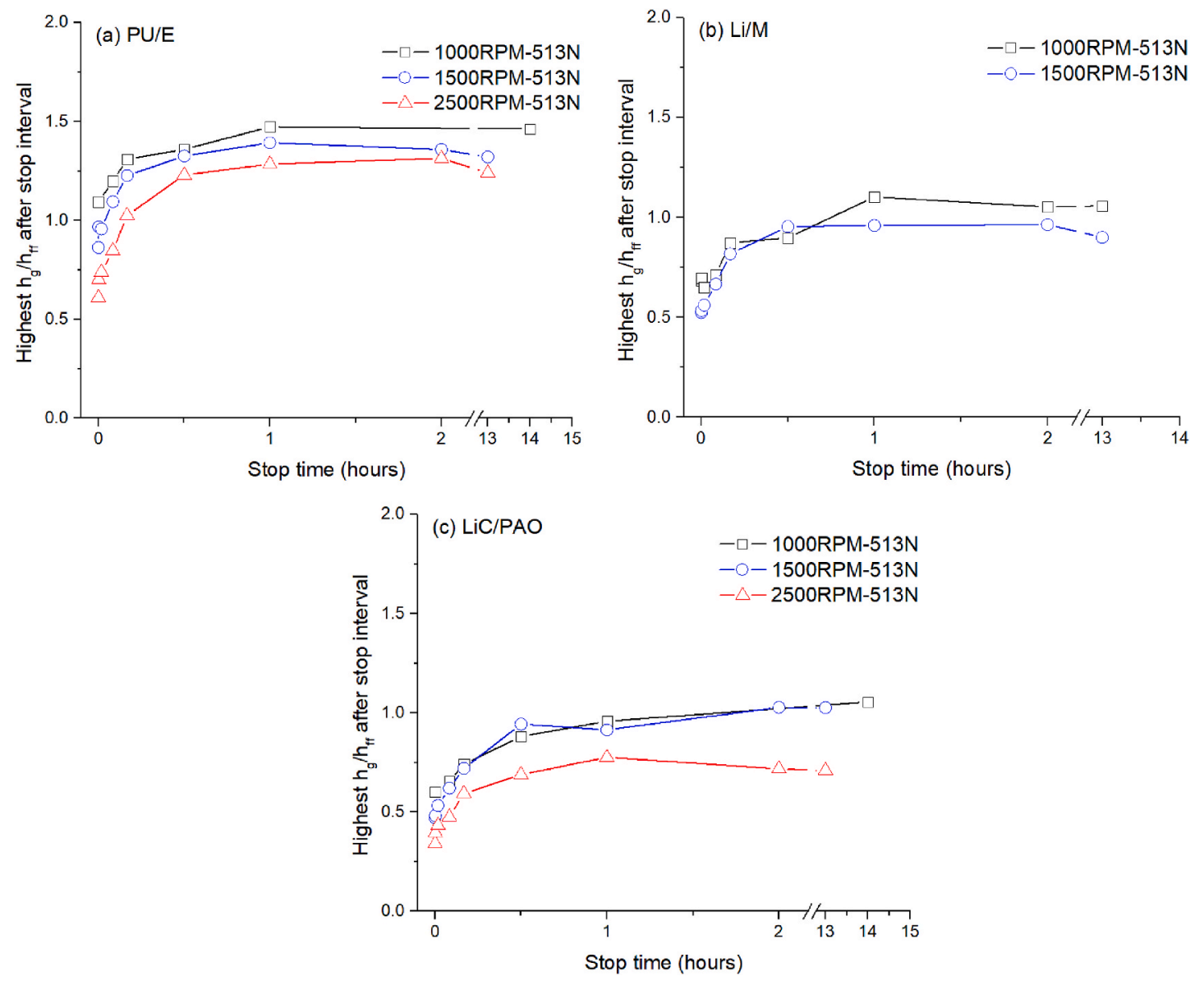

Fig. 4. Normalized film thickness versus stop time for various speeds with test greases of (a)PU/E; (b)Li/M; (c)LiC/PAO.

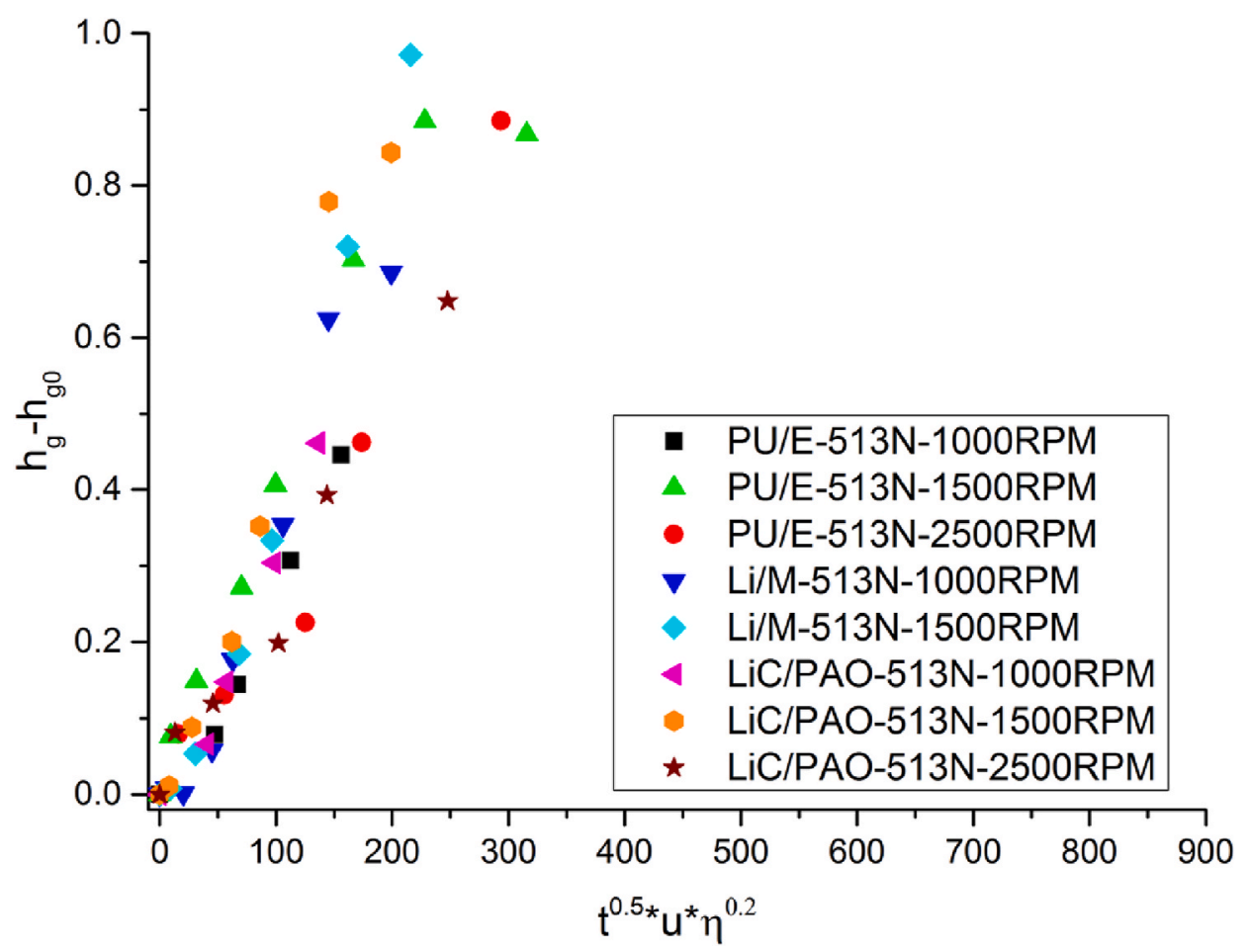

Fig. 5. Increase in film thickness due to stops as a function of stop duration (s), mean entrainment speed for the inner ring-ball contacts (m/s) and base oil viscosity for an axial load of $513 \mathrm{~N}$ and a $6209-2 \mathrm{Z}$ bearing. 


\subsection{Varying load}

Film thickness measurements with the greases PU/E and LiC/PAO were performed with a fixed speed of $2500 \mathrm{rpm}$ and at loads of 513, 700 and $900 \mathrm{~N}$. Fig. 6 shows the development of the highest normalized thickness of lubricating film during different stop durations at different loads. Similar to the results with different speeds, the normalized film thickness $h_{g} / h_{f f}$ increases with increasing stop-time until reaching a stable value (saturation). Again replenishment occurred during the stoptime. The increase of load leads to less replenishment during an identical stop-time for the PU/E grease. For LiC/PAO grease, the effect of load is very small.

Plotting the normalized film thickness is very instructive because it shows the level of starvation. However, as in the previous section, to investigate replenishment itself, it is more transparent to plot the film thickness directly. This is done in Fig. 7.

The increase in thickness of the lubricating film can be calculated by:

$\Delta h \sim \frac{t^{1 / 2} u^{1.2}}{\eta^{0.2} a_{y}}$

A varying load leads to a varying Hertzian contact half width $a_{y}$. The impact of contact width is very large compared to the other parameters (speed and base oil viscosity). The width over which replenishment needs to take place is apparently dominating.

\subsection{Varying rotating direction}

$\mathrm{PU} / \mathrm{E}$ and LiC/PAO were selected to study the impact of a change in the rotating direction on the grease film thickness. Other than in the previous sections where the speed of the bearing was put to zero, in this section it will be reversed. Each test was done with a fixed speed of 2500 rpm and load of $513 \mathrm{~N}$. The test procedure was as follows: first the bearing was started with fresh grease and rotated in anti-clockwise direction for a long enough time to achieve a quasi-static film thickness. Next the bearing was stopped for $1 \mathrm{~h}$ and restarted running in clockwise direction for $1 \mathrm{~h}$. Finally, the bearing was stopped again for some time (13 $\mathrm{h}$ for PU/E and $2 \mathrm{~h}$ for LiC/PAO) and restarted, running in anticlockwise direction.

Fig. 8 and Fig. 9 show the results for PU/E and LiC/PAO, respectively. In Fig. 8 the bearing was run for $6 \mathrm{~h}$, after which the normalized film thickness $h_{g} / h_{f f} \approx 0.6$. The bearing was then stopped and the film thickness became about $h_{g} / h_{f f} \approx 0$. The bearing was then standing idle for $1 \mathrm{~h}$ and track replenishment could take place, such that after starting up again, but now in counter rotation, the normalized film thickness $h_{g} /$ $h_{f f} \approx 1$.4. During running for $1 \mathrm{~h}$ the film thickness decreased to the quasi steady state value of $h_{g} / h_{f f} \approx 0.6$. The bearing was then stopped for $13 \mathrm{~h}$ during which the film thickness was zero again and track replenishment could take place. When the bearing was started up in counter rotation the film thickness behaved similar as before: initially a thick film $h_{g} / h_{f f}$ $\approx 1.4$, which decreases due to starvation. Fig. 9, for LiC/M grease shows similar behavior.

Figs. 8(b) and Fig. 9(b) show the normalized film thickness after starting up the bearing after standing still for a certain "stop time". The black points have been taken from the results from the previous section, i.e., running always in the same direction, whereas the black points show the values in the case of counter rotation as described in this section. The PU/E grease results are exactly the same for the two situations. For the LiC/M grease there is only a small difference which can be ascribed to the inaccuracy of the test.

Therefore, the impact of speed direction change on the initial normalized thickness after a stop interval can be neglected.

\section{Discussion}

Grease lubricated sealed and greased for life ball bearings are mainly working under starved lubrication conditions. It was revealed in our earlier paper [3] that, for pure axial load, the normalized thickness of the lubricating film was determined by the product of viscosity of the base oil, bearing speed and width of the contacts. The thickener also plays a role, however, only at very low speeds and during start-up. Actually, the film thickness itself does not depend very much on speed under starved lubrication conditions.

Of course bleed plays an important role as well. However, this is not "measurable" during the first, say, $24 \mathrm{~h}$ of running. Bleed, will provide the contacts with oil over very long times and is therefore important for long running times, to get a long grease life.

There is much speculation on film thickness for bearings lubricated with grease and are working under non-constant conditions, with extremes such as start-stops. After all, in practice calculations are based on steady state conditions. This paper shows that the film thickness evolution during start stops is more favorable than can be expected based on calculations or measurement results done for steady state. It is shown here that a stop will give time for additional track replenishment leading to an initially thicker film than may be expected.

It was possible to describe this effect in a single curve containing only the stop time, base oil viscosity, speed and contact width. This suggests that these are the only (or most) relevant parameters for track replenishment. Hence, track replenishment during standstill is determined by the same parameters as those during running. This does not mean that other parameters such as for example surface tension are not important. However, since such a parameter does not vary much for "standard" lubricating oils, it does not appear as important.

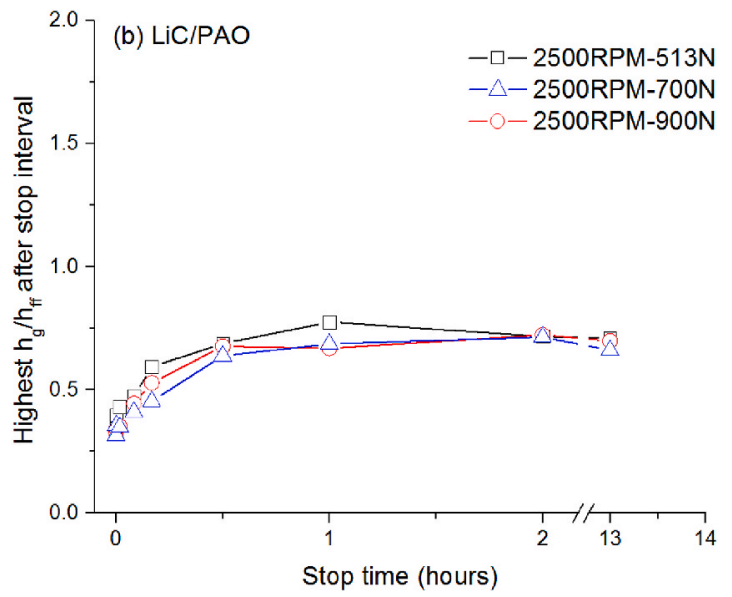

Stop time (hours)

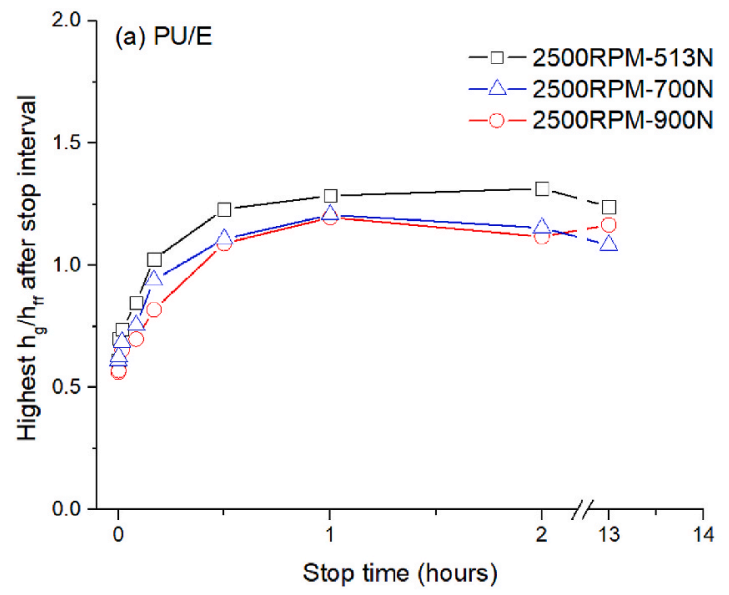

Fig. 6. Development of highest normalized film thickness with load after each stop duration: (a)PU/E; (b)LiC/PAO. 


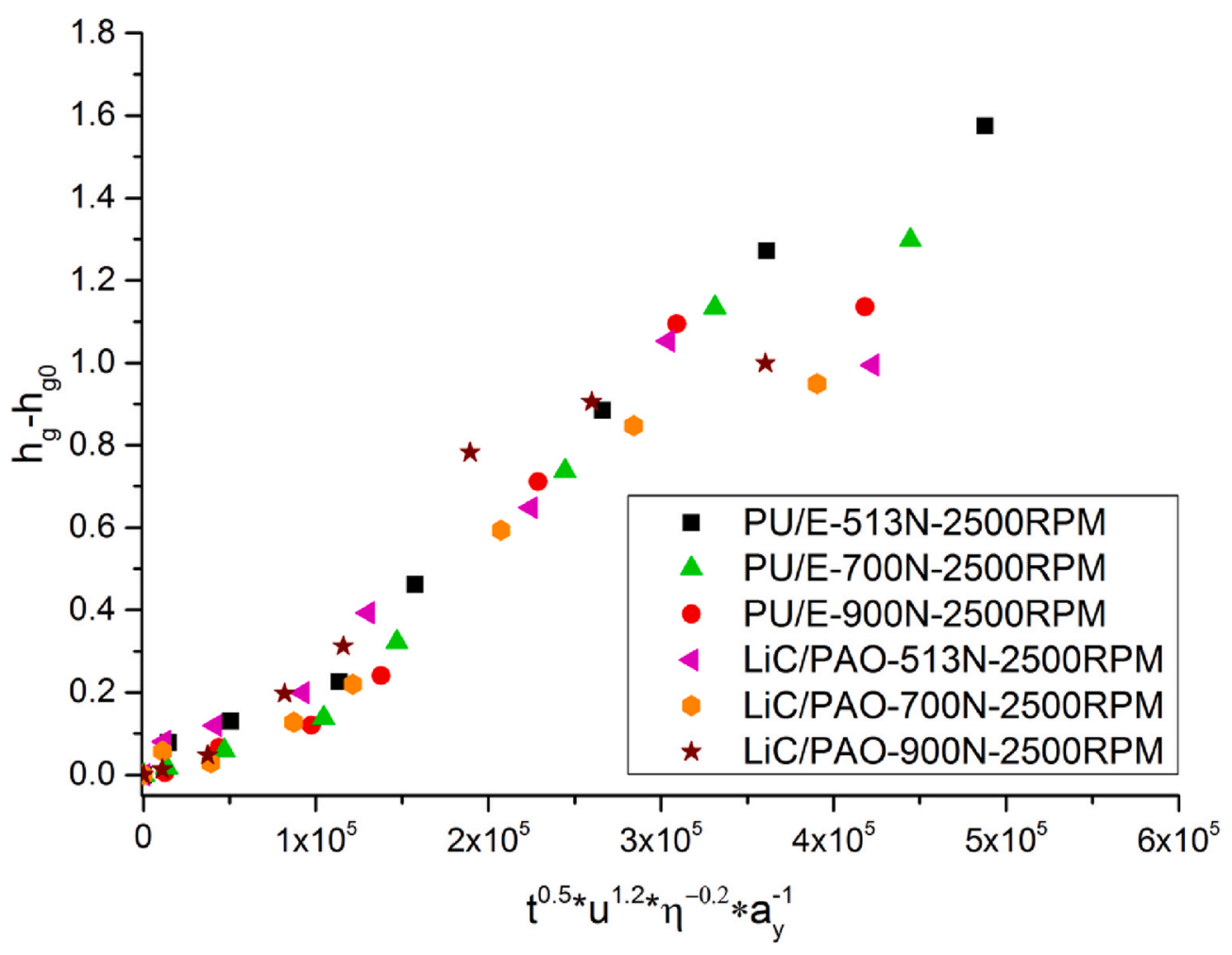

Fig. 7. Increase in film thickness by stopping the bearing for a time t. 6209-2Z bearing running under pure axial load conditions.
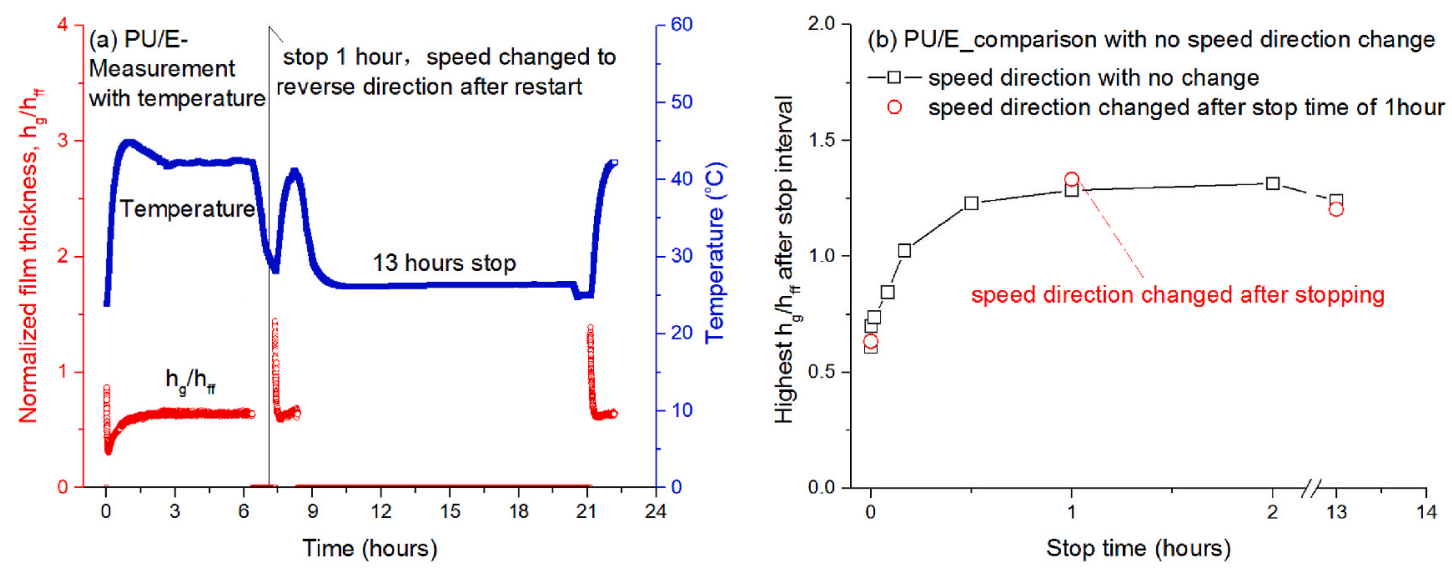

Fig. 8. Impact of rotation direction change on the thickness of lubricating film for PU/E: (a) film thickness change with temperature; (b) comparison with no change in rotation direction.

Track replenishment during stand still is determined by the same parameters as those that determine the film thickness, but with a different significance. It is apparent that the contact width plays a very large role: wide contacts need more time for replenishment than narrow contacts. The rationale behind the exponents in the track replenishment equation could not be given here. Track replenishment happens around the contacts by capillary flow and between the contacts, driven by surface tension. The first effect is much faster than the second effect. One may expect therefore two regimes in the replenishment plot, Fig. 6. The accuracy of our experiments was not high enough to distinguish this.

It may look surprising that the speed is significant here. This is most likely the result of the effective length between two balls $u \times t$ and the impact of speed on thickness of lubricating film. The effective length can be translated into an effective time $s / u$, with $s$ the distance between two balls. This effective time is the time between over-rolling. This means that during start-up at higher speeds more volume is available than in the case of lower speeds, hence less starvation and thicker films. The second effect that plays a role is that in the case of starvation, i.e. before the stop, the thickness of lubricating film hardly depends on bearing speed whereas the film thickness is proportional to $u^{0.7}$ under fully flooded lubrication regime. After start up the contacts are less starved than before a stop. The exact contribution of these effects cannot be given here. Model development on capillary flow and migration should give an answer to this. However, this goes beyond the scope of this paper and is a topic for further research.

\section{Conclusions}

The semi steady state film thickness in an axially loaded grease lubricated bearing does not depend very much on speed. However, the thickness of lubricating film at very low speeds could be attributed to the thickener as well. The bearing contacts are mainly experiencing starved 

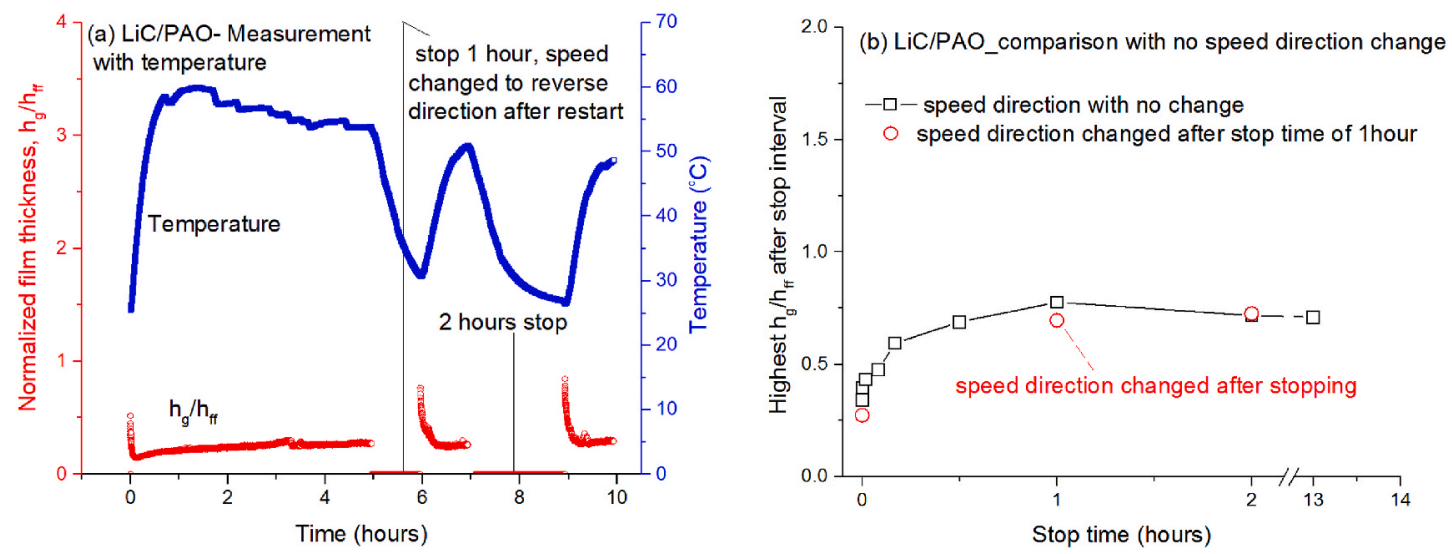

Fig. 9. Impact of rotation direction change on the thickness of lubricating film for LiC/PAO: (a) film thickness change with temperature; (b) comparison with no change in rotation direction.

lubrication conditions. During a stop the thickness of lubricating film quickly goes to zero thickness due to the absence of hydrodynamic effects. While the bearing is not rotating, track replenishment takes place such that the film thickness is unexpected large when the bearing is started up again. Clearly, during start-up, after a stop, the contacts are running less starved than before the stop. This effect is favorable for grease lubricated bearings although it should be noted that the duration where the films are higher are very short. Thick films during start-up are obtained mainly by stopping sufficiently long, under low load conditions where the contacts are narrow. The effect is more pronounced at higher speeds than at lower speeds.

The increase in thickness of lubricating film can be calculated by:

$\Delta h \sim \frac{t^{1 / 2} u^{1.2}}{\eta^{0.2} a_{y}}$

showing that stationary replenishment is dominated by the contact width and bearing speed. This increase in thickness of lubricating film is dominated by replenishment. The fact that the exponents in this equation are different from those that determine the film thickness during continuous rotation suggests that the replenishment mechanism during a stop is different from that during running.

The direction of speed when starting up does not have an effect on this. In other words, for the thickness of the lubrication film in the EHL regime, oscillating motion, with sufficiently long time between a change in the direction of speed (so we are not referring to small oscillations) can be described by a unidirectional start-stop sequence.

\section{CRediT authorship contribution statement}

Hui Cen: Conceptualization, Methodology, Experiments, Data processing, Writing - original draft, Writing - review \& editing. Piet M. Lugt: Conceptualization, Data processing, Supervision, Writing - review \& editing.

\section{Declaration of competing interest}

The authors declare that they have no known competing financial interests or personal relationships that could have appeared to influence the work reported in this paper.

\section{Acknowledgement}

This study was performed at Universiteit Twente, the Netherlands. The authors would like to thank the technical support from R.J. Meijer of Universiteit Twente and SKF Research and Technology Development for permission to publish this paper. The authors also would like to thank the financial support by Natural Science Foundation of Henan Province, China (Grant No. 212300410387) and National Natural Science Foundation of China (Grant No. 51905463).

\section{Notation}

$h_{g} \quad$ Measured film thickness [nm]

$h_{f f} \quad$ Fully flooded base oil film thickness (Hamrock Dowson) [nm]

$d_{a} \quad$ Outer diameter of test bearing [mm]

$d_{m} \quad$ Bearing pitch diameter [mm]

$d_{i} \quad$ Diameter of inner raceway groove in the test bearing [mm]

$d_{b} \quad$ Bore diameter of test bearing [mm]

$d_{0} \quad$ Diameter of outer raceway groove in the test bearing [mm]

$d \quad$ Ball diameter of test bearing [mm]

$R_{x} \quad$ Reduced radius in $x$ direction (rolling) [mm]

$R_{y} \quad$ Reduced radius in $y$ direction (transverse to rolling) [mm]

$t \quad$ Stop duration [s]

$u \quad$ Linear speed $[\mathrm{m} / \mathrm{s}]$

$\eta \quad$ Base oil dynamic viscosity at the ambient temperature $[\mathrm{Pa} \cdot \mathrm{s}]$

$\Delta h \quad$ The increase in film thickness after restart [nm]

$h_{g 0} \quad$ Film thickness before the stop [nm]

$a_{y} \quad$ Elliptical geometry [mm]

\section{References}

[1] Hamrock BJ, Dowson D. Elastohydrodynamic lubrication of elliptical contacts for materials of low elastic-modulus I - fully flooded conjunction. Trans ASME, J Lubr Technol 1978;100:236-45. https://doi.org/10.1115/1.3453152.

[2] Nijenbanning G, Venner $\mathrm{CH}$, Moes H. Film thickness in elastohydrodynamically lubricated elliptic contacts. Wear 1994;176:217-29. https://doi.org/10.1016/ 0043-1648(94)90150-3.

[3] Cen H, Lugt PM. Replenishment of the EHL contacts in a grease lubricated ball bearing. Tribol Int 2020;146:106064. https://doi.org/10.1016/j. triboint.2019.106064.

[4] Wedeven LD, Evans D, Cameron a. Nasa technical Nasa T M X-52869 optical analysis of ball bearing starvation. 1970.

[5] Chiu YP. An analysis and prediction of lubricant film starvation in rolling contact systems. ASLE Trans 1974. https://doi.org/10.1080/05698197408981435.

[6] Wen SZ, Ying TN. A theoretical and experimental study of EHL lubricated with grease. J Tribol 1988;110:38. https://doi.org/10.1115/1.3261572.

[7] Chevalier F, Lubrecht AA, Cann PME, Colin F, Dalmaz G. Film thickness in starved EHL point contacts. J Tribol 1998;120:126-33.

[8] Jacod B, Pubilier F, Cann PMM E, Lubrecht AAA. An analysis of track replenishment mechanisms in the starved regime. Lubr Front 1999;36:483-92. https://doi.org/10.1016/s0167-8922(99)80069-8.

[9] Cann P, Lubrecht AA. Analysis of the mechanisms of grease lubrication in rolling element bearings. Lubric Sci 1999;11:227-45. https://doi.org/10.1002/ ls.3010110303.

[10] Cann PM, Lubrecht AA. The effect of transient loading on contact replenishment with lubricating greases. In: Tribol. Ser, vol. 43. Elsevier; 2003. p. 745-50.

[11] Damiens B, Lubrecht AA, Cann PM. Influence of cage clearance on bearing Lubrication@. Tribol Trans 2004;47:2-6. https://doi.org/10.1080/ 05698190490279128. 
[12] Cann PME, Lubrecht AA. Bearing performance limits with grease lubrication: the interaction of bearing design, operating conditions and grease properties. J Phys D Appl Phys 2007;40:5446-51. https://doi.org/10.1088/0022-3727/40/18/S05.

[13] Damiens B, Venner CH, Cann PME, Lubrecht AA. Starved lubrication of elliptical EHD contacts. J Tribol 2004;126:105, https://doi.org/10.1115/1.1631020.

[14] van Zoelen MT, Venner CH, Lugt PM. Free surface thin layer flow on bearing raceways. J Tribol 2008;130:021802. https://doi.org/10.1115/1.2805433.

[15] van Zoelen MT, Venner CH, Lugt PM. Free surface thin layer flow in bearings induced by centrifugal effects. Tribol Trans 2010;53:297-307. https://doi.org/ 10.1080/10402000903283284.

[16] Venner CH, Van Zoelen MT, Lugt PM. Thin layer flow and film decay modeling for grease lubricated rolling bearings. Tribol Int 2012;47:175-87. https://doi.org/ 10.1016/j.triboint.2011.10.019.

[17] Svoboda P, Kostal D, Krupka I, Hartl M. Experimental study of starved EHL contacts based on thickness of oil layer in the contact inlet. Tribol Int 2013;67: 140-5. https://doi.org/10.1016/j.triboint.2013.07.019.

[18] Wikström V, Jacobson B. Loss of lubricant from oil-lubricated near-starved spherical roller bearings. Proc Inst Mech Eng Part J J Eng Tribol 1997;211:51-66. https://doi.org/10.1243/1350650971542318.
[19] Zhou Y, Bosman R, Lugt PM. An experimental study on film thickness in a rolling bearing for fresh and mechanically aged lubricating greases. Tribol Trans 2019: 1-10. https://doi.org/10.1080/10402004.2018.1539202. 0.

[20] Cen H, Lugt PM. Film thickness in a grease lubricated ball bearing. Tribol Int 2019; 134:26-35. https://doi.org/10.1016/j.triboint.2019.01.032.

[21] Hamrock BJ, Dowson D. Elastohydrodynamic lubrication of elliptical contacts for materials of low elastic modulus i-fully flooded conjunction. J Tribol 1978;100: 236-45. https://doi.org/10.1115/1.3453152.

[22] Gershuni L, Larson MG, Lugt PM. Lubricant replenishment in rolling bearing contacts. Tribol Trans 2008;51:643-51. https://doi.org/10.1080/ 10402000802192529.

[23] Lugt PM. Grease lubrication in rolling bearings. John Wiley \& Sons; 2012.

[24] Miner MA. Cumulative fatigue damage. J Appl Mech 1945;12:A159-64.

[25] Heemskerk RS, Vermeiren KN, Dolfsma H. Measurement of lubrication condition in rolling element bearings. ASLE Trans 1982;25:519-27. https://doi.org/10.1080/ 05698198208983121.

[26] Wilson AR. The relative thickness of grease and oil films in rolling bearings. Proc Inst Mech Eng 1979;193:185-92. https://doi.org/10.1243/PIME_PROC_1979_193_ 01902.

[27] Walther C. The evaluation of viscosity Data. Erdol und Teer 1931;7:382-4. https:// doi.org/10.1016/j.(73. 